\title{
DBaaS Using HL7 Based on XMDR-DAI for Medical Information Sharing in Cloud
}

\author{
Ho-Kyun Park ${ }^{1}$ and Seok-Jae Moon ${ }^{2}$ \\ ${ }^{1}$ School of IT Convergence Engineering, Shinhan University, Gyeonggi, Korea \\ ${ }^{2}$ Department of Computer Science, Kwangwoon University, Seoul, Korea \\ hkpark@shinhan.ac.kr,msj8086@kw.ac.kr
}

\begin{abstract}
In recent, a medical information system that enables hospitals with different hospital systems to efficiently share and exchange medical information is demanded. For transferring medical information between medical information systems, the connection course that transforming medical data in compliance with a HL7 of the medical information exchange protocol is required. This study suggests DBaaS that efficiently cooperates the schema structure between each different medical information system by using XMDR-DAI for different HL7 standard protocol. Furthermore, this study applied $X M D R-D A I$ in order to solve the collision due to schema heterogeneity that occurs in the information connection course between medical systems.
\end{abstract}

Keywords: HL7, Cloud, XMDR-DAI, Interoperability, schema heterogeneity

\section{Introduction}

Recently, the informatization in the medical area has been rapidly made. Medical informatization results in many changes to the medical system more than easily sharing and utilizing plenty of information [1]. In particular, in case that an emergency patient is transferred to a general hospital for an operation after diagnosis in a small hospital, the delay of diagnosis due to repetitive emergency diagnosis can be prevented when the basic test information made during transfer to the general hospital is transmitted to the general hospital and the patient condition is understood in advance before patient's arrival at the hospital for preparing an operation. Like this, studies on the standardization for sharing information of medical treatment are required to solve the problems of time and space. When exchanges of medical data between different hospital information systems are required, even a hospital that treats affairs by department unit often uses various programs developed by different companies. Accordingly, data exchanges between diverse programs without compatibility are required and at this point, HL7 (Health Level Seven) [2-3] was suggested as a standard to correctly exchange data between different medical institutions.

This study suggests the cloud based DBaaS [4] in which medical data between hospital systems can be shared in compliance with the HL7 type using XMDR-DAI [5] for medical data according to the protocol defined in the HL7 standard. This DBaaS has the characteristic to store and cooperatively share medical information based on the HL7 message required for interchange between hospitals through each mapping of medical information of different types. Moreover, it can mutually integrate medical information, and create individual medical information schema, and share individual medical information with other hospitals through a broker server. The composition of this study is as follows. Chapter 2 describes related studies, and Chapter 3 explains the suggested DBaaS composition and internal flow. Chapter 4 describes the HL7 mapping agent structure, and Chapter 5 explains XMDR-DAI. The last Chapter 4 describes results and future research. 


\section{Related Works}

HL7 is a standard protocol to exchange data between health and medical information systems [6-7]. At present, it is the standard protocol of North America for electronic exchanges of medical information. The HL7 message is composed of segment, field and component.

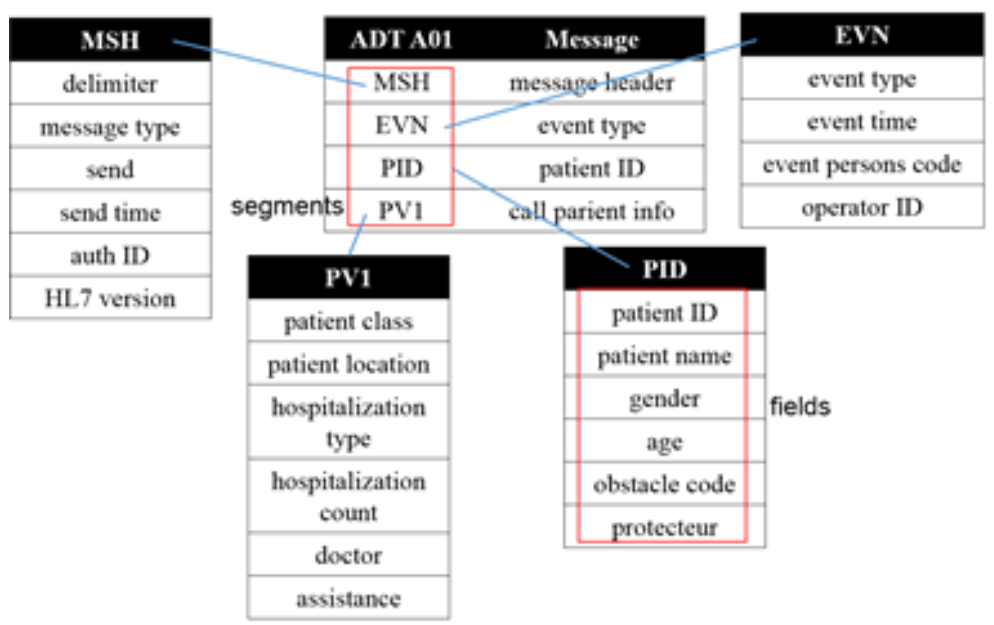

Figure 1. HL7 Protocol Message Structure

Figure 1 is the structure of the HL7 message. This message is the smallest unit of data transfer and composed of segments. The message starts with Message Header Segment $(\mathrm{MSH})$ and divides messages by message type and trigger event. Figure 1 shows the message structure, and the message is composed of segments, and the segment consists of fields. A segment is a logical gathering of fields in fixed order. There are segments left for the essential segment, selective segment and downward compatibility. An essential segment is a segment that must be filled within a message. A selective segment is not used or allows many repetitions. The selective segment is indicated as [segmentID], and the repetitive segment as \{segmentID\}. A segment can be distinguished by the 3 initial letters of segment ID. A segment is divided by a segment separator (normally $\langle\mathrm{CR}\rangle$ ). In Figure 1, there are composed of MSH standing for a message header, EVN describing an event, PID representing the ID of a patient and PV1, the visit information of a patient. It has a type that has additional information following the above. For example, they are information on test results, performance and estimate. The actual contents of the message are expressed in the field of the segment. A field is a gathering of components and a component is composed of subcomponents. A field is defined as a variety of data such as letter, number, date, time and mixed sentence, and it is transferred as a type of a string. The maximum length property of a field defines the maximum letter number that can be expressed in a field. The field allows a null value. The field allows repetition. Special letters used for composing messages are a segment separator, field separator, component separator, subcomponent separator and escape letter.

\section{The DBaaS Architecture}

\subsection{Proposed System Overview}

The system suggested by this study is the same as Figure 2. Medical information systems used by each hospital interwork with Coordinator by using Hospital DB based on the HL7 protocol. Coordinator provides interwork information with each medical information systems through Hospital Category DB and enables exchanges of medical 
information through Data Hub. When medical information required by a hospital is demanded to Coordinator, it searches for a hospital and patient information through a previously registered original key. Medical data of each hospital system is operated after mapping in compliance with the HL7 protocol. Below is the explanation on components of this system.

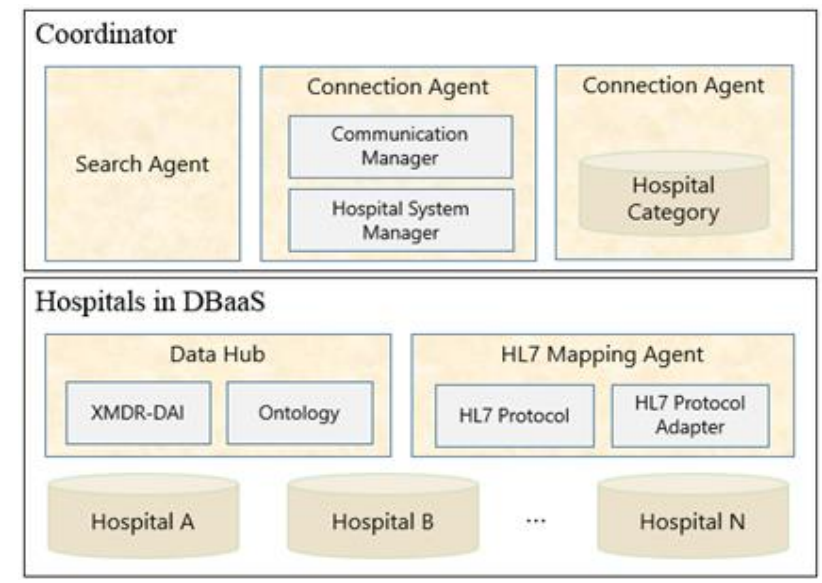

Figure 2. The Proposed DBaaS Based on XMDR-DAI

- Coordinator: This class makes each physical hospital information as category key value, and connects different hospitals, and mutually searches for medical data, and is composed of Search Agent, Connection Agent, and Hospital Category DB.

Search Agent: This agent interworks medical data required by each hospital with Connection Agent and supports swift search.

Connection Agent: This agent deals with a demand to connect medical system of each hospital. When a connection demand is received through Communication Manager, Hospital System Manager starts connecting and searching through a category key value.

- Hospitals DBaaS: This class provides handling of a medical data demand of each hospital by using the HL7 protocol message through mapping, and is composed of Data Hub, HL7 Mapping Agent, and Hospitals DBs.

Data Hub: It enables medical information stored in each hospital DB to be connected. At this moment, handling of schema collision that can occurs between medical data is solved by XMDR-DAI and Ontology.

HL7 Mapping Agent: It provides the results as a file type from mapping medical information data through each HL7 protocol message and Adapter.

\subsection{System Flow}

Figure 3 is the whole process that interoperates medical information between medical information systems that use DBaaS suggested by this study. As seen Figure 3, Hospital A requests connection of the medical information data of Hospital B through the Connection Agent. Then, Search Agents search for Hospital B by using Hospital Category DB and use the HL7 protocol message through HL7 Mapping Agent and acquire necessary information. Then, Data Hub searches for requested information from the operating DB of Hospital B in XMDR-DAI and confirms the possibility of collision and performs queries on the requested information. After its performance, the results requested by Search Agent are rendered to Hospital A. 


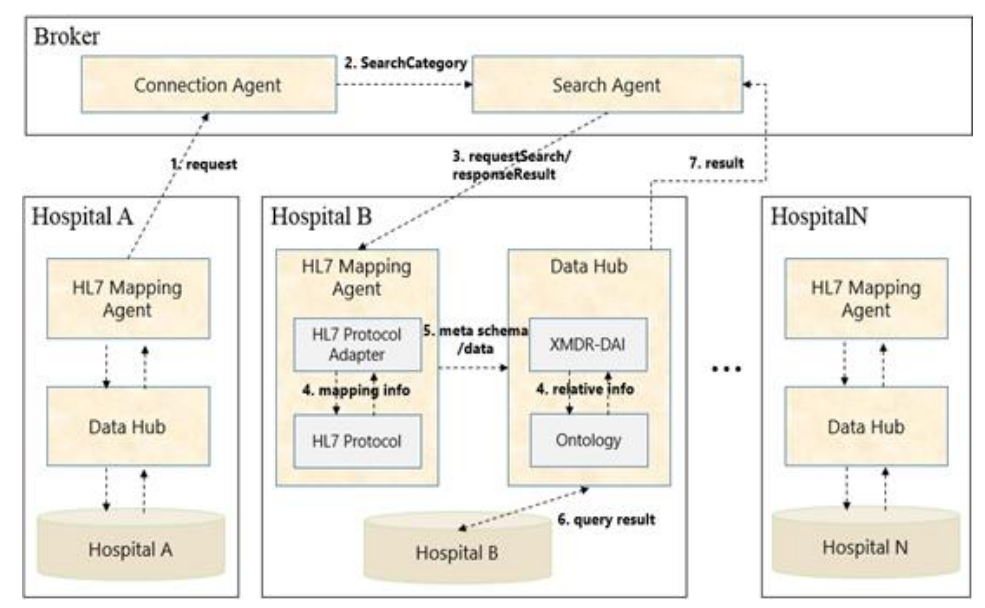

Figure 3. System Flow of DBaaS based on XMDR-DAI

\section{HL7 Mapping Agent}

\subsection{HL7 Protocol Adapter}

The HL7 Protocol Adapter of this DBaaS implemented the User HL7 Agent that transforms information according to the coding rules of HL7, the standard method in order to transfer patient registration and test information occurring in the client system to a hospital server system. In addition, this Agent implemented parts necessary for a hospital server system that receives from a client system a message transformed from the encoding rules in compliance with XMDR-DAI to send a message of successful receipt. The transmitting and receiving course of a message occurring in the HL7 Agent is the same with Figure 4. When a user sends the message of patient registration and test information, a message is created by the HL7 Protocol based on XMDR-DAI in the HL7 Agent, and the created message is transferred to a hospital system engine by using TCP/IP Protocol. The transferred HL7 message passes through integrity verification and decoding, and a message to confirm successful receipt is sent to the User HL7 Agent.

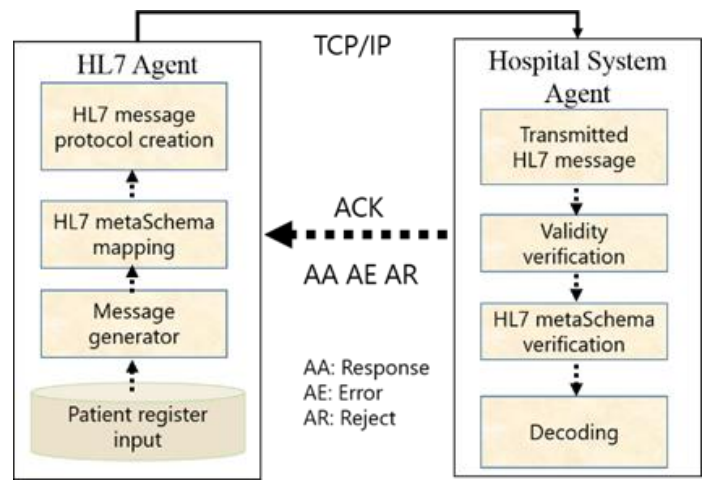

Figure 4. HL7 Adapter Message Send/Receive Flow

\subsection{HL7 Protocol Generator}

Basic medical information requires an encoding course that transforms the information to XMDR-DAI based HL7 message for exchanges between medical information systems. HL7 Standard 2.4 Version exchanges information between medical systems as a message of the ASCII text type. This generator changes it to the HL7 message to be sent by using basic data. On the grounds that the generator has the message structure and segment 
information, an abstract message structure is transformed and represented according to HL7 message creation rules.

\subsection{HL7 Protocol Parser}

The HL7 Protocol Parser plays a role in extracting information on field, component and subcomponent in text to process a message in its system when the message is received from other medical information system that progress a message of HL7 2.x.

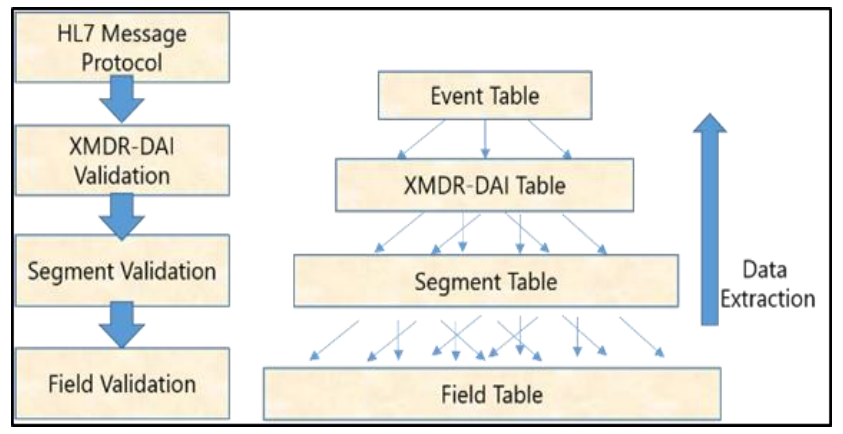

Figure 5. HL7 Protocol Parser Function

Figure 5 divides the HL7 message into segment unit and field unit again to extract event tables and data according detailed items respectively. The segment and its subfield included in one event are understood successively according to the existence and repetitive type specified in event items, and verification work on detailed items is performed simultaneously. In Figure 5, a segment that is essential among the transferred messages but missing or out of order is tested in the segment verification process. In Figure 5, the demand file, field length, data type, component and subcomponent are tested in the field verification process. Required fields are classified as essential, selective and unused according to the selection of fields in segments. It tests whether the field value of an essential field is null or the field does not exist. The length of a field defines the maximum length of each field in the HL7 standard. It tests whether a length is longer than the length defined in the standard. The data type has 55 data types in the HL7 standard. Each field has a defined data type and satisfaction with this type is tested. In some fields, the data values are defined to have the values in only the fixed tables. For instance, the sex code can only have M (Male), F (Female) and U (Unknown). The component and subcomponent can have many components in one field, and one component can have several subcomponents. As each component or subcomponent has a data type, value and length like a filed, a test of subcomponent is required.

\section{XMDR-DAI Composition}

\subsection{HL7 Based on XMDR-DAI}

This Chapter applied XMDR-DAI [5] for mapping between HL7 protocol schema information. Figure 6 shows the solution to schema collision occurring in the process sharing HL7 based medical data between medical information systems. Figure 6 is composed of the Ontology Thesaurus area consisting of MSO (Metadata Semantic Ontology) and InSO (Instance Semantic Ontology), and Meta Global Schema and Meta Local Schema. The Meta Global Schema (Global Schema) shows the schema table structure necessary for sharing medical data and is composed of each table (Patient, Palnfo) and field. The Metal Local Schema (Local Schema) shows the structure of tables and fields in the medical information database participating in this application system. 


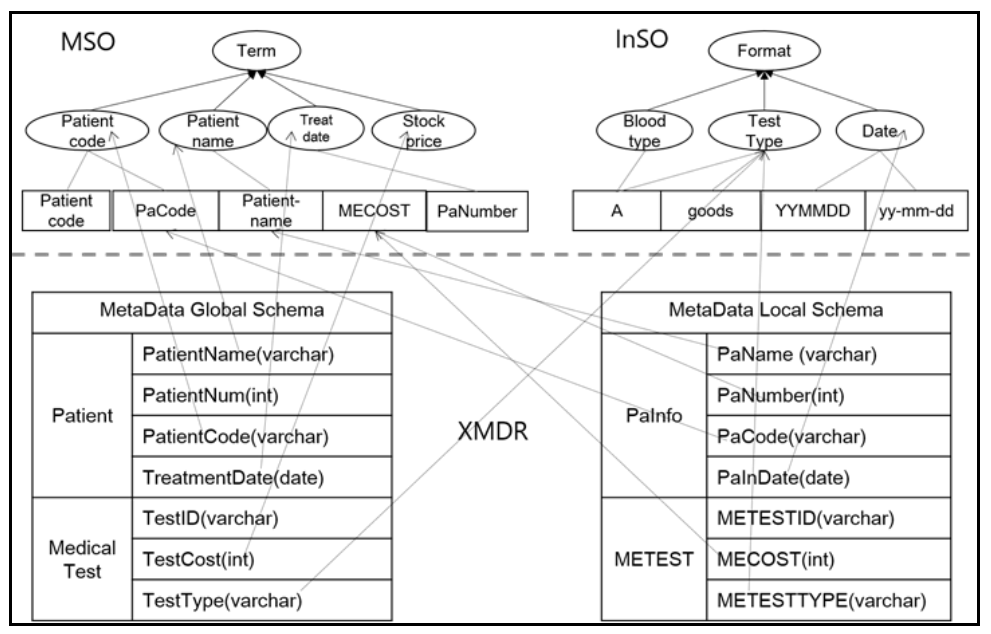

Figure 6. XMDR-DAl for HL7 Schema

For example, information in the Local Schema matching the field name of 'PatientName' of the 'Patient' table in the Local Schema is searched through MSO. It is found that in the MSO Map, the 'PatientName' node is divided into the 'Patient-name' and among them, the 'Patient-name' is the 'PaName' in the 'Palnfo' table of the Local Schema. Through this matching method, it can be transformed by finding things with the same meaning between the Global Schema and Local Schema. In the InSO map, the collision in data unit and expression is solved. For example, it is found that in the 'TestType' of the Global Schema, the 'Type' is the varchar type and the 'TestType' node is divided into 'A', 'Good.' Through this matching method, code, unit and serial number types can be transformed in the data expressions between the Global Schema and Local Schema.

\subsection{XMDR-DAI Agent}

Figure. 7 shows HL7 based on XMDR-DAI operating algorithm where itemLists and handle are entered at Input. Once entered, handle is divided into 0, 1, 2, and 3, schema information of table, field, value, and loc at XMDRExtract() is extracted and returned. If table(0), field(1), value(2) are delivered at handle, Ontology Agent is perceived to call, and if table(0), field(1), value(2), and $\operatorname{loc}(3)$ are delivered at handle, Local DataHub is perceived to call, with relevant schema information being returned. 


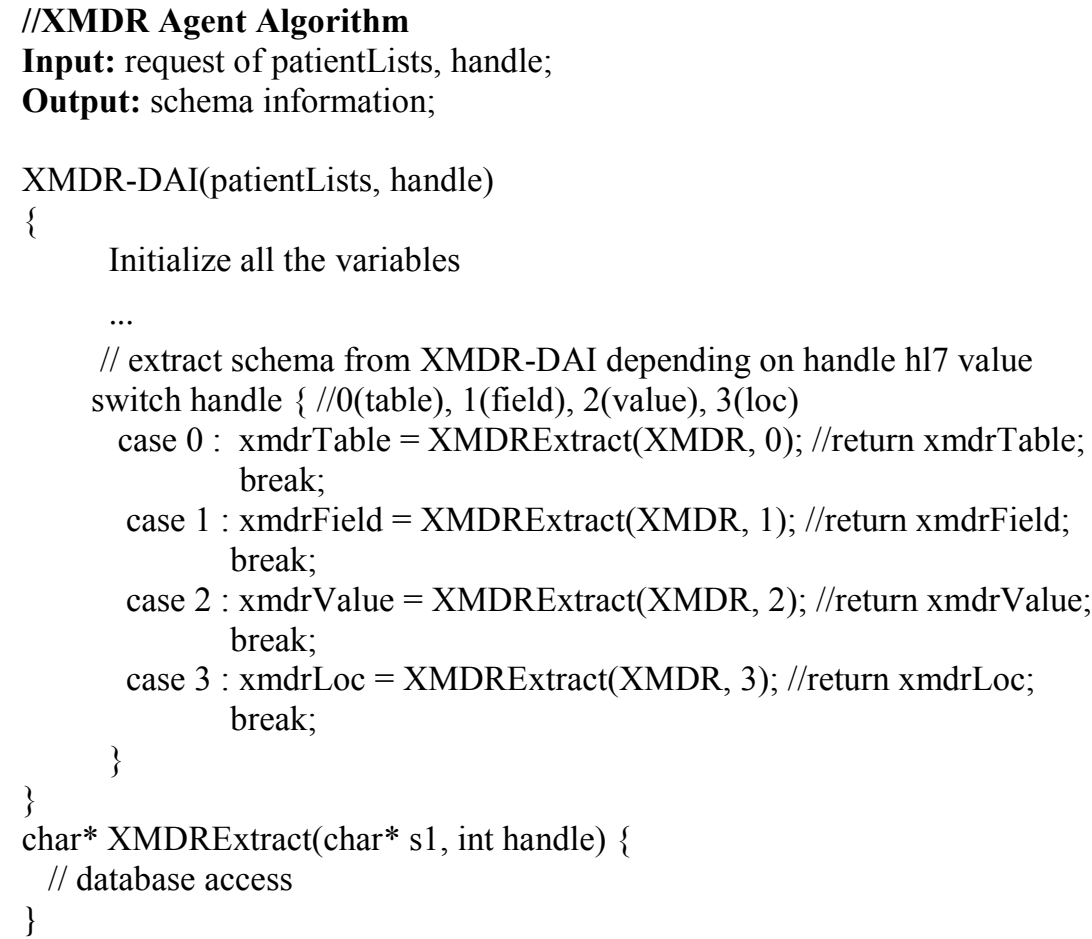

Figure 7. XMDR-DAl Agent Algorithm

\subsection{XMDR-DAI Agent Analysis}

This Chapter evaluates the function based on the performance results of collection service as well as the access service of medical data by applying XMDR-DAI in the exchange and performance process of the HL7 message. In the process performed according to the procedure stages of process in the medical system, the normal and reverse processes are performed, and the reverse process is excluded in the objects of analysis. In addition, connectivity filtering in the Ontology Thesaurus of XMDR-DAI is not over 20 semantic connectivity conditions in the query. Figure 8 evaluates the function of the performance process time about the performance procedure number inside of HL7 message exchanges applying XMDR-DAI. For the test to evaluate the function, the database servers of 4 cooperative medical systems were used, and patient medical information on collection results including over around 15,000 cases was utilized. Furthermore, the number of the system internal performance procedures about the medical process was not over 9 stages at maximum. Like Figure 8, when medical cooperative information is performed according to the medical information integrating method applying MDM (Master Data Management), that applying GDS (Grid Data Service) and this XMDR-DAI method, it is comparison of function according to each performance procedure number. The internal procedure of one process from stage 1 to 4 all showed a process time from $1100 \mathrm{~ms}$ to $1500 \mathrm{~ms}$, but it rapidly increased the process time from $2400 \mathrm{~ms}$ to $6600 \mathrm{~ms}$ from over stage 5. However, the MDM and XMDR-DAI methods showed a difference of around $1000 \mathrm{~ms}$ from stage 7 more than the GDS method. In the result of this process time, because GDS is a query method based on the Meta Data Schema, and the MDM and XMDR-DAI methods are a query method based on the Master Data, there is a little difference. But, given that the three methods showed the increase of the process time from stage 4 , the cooperative process internal procedures based on the HL7 message is efficient at a four- stage level. Therefore, the suggested XMDR-DAI based system should consider it when a medical process by cooperation is defined. As the number of process procedures increase, because medical information 
cooperation itself becomes complicated, it is realized that the suggested cooperative system needs to perform a process at a four-stage level.

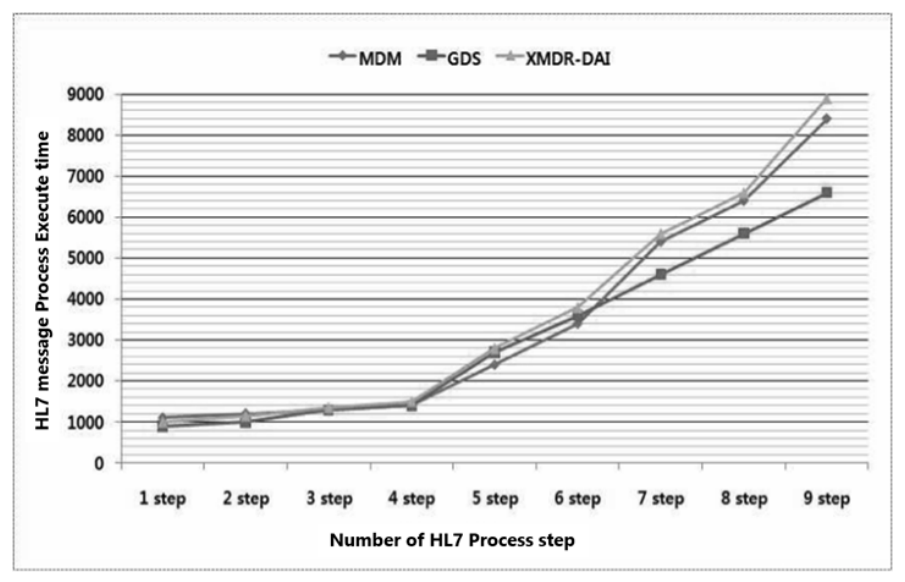

\section{Figure 8. Perform HL7 Message Exchange Inside Apply XMDR-DAI Carried Out for the Number of Steps}

\section{Conclusion}

This study suggests the cloud based DBaaS in which medical data between hospital systems can be shared in compliance with the HL7 type using XMDR-DAI for medical data according to the protocol defined in the HL7 standard. The suggested system implemented the DBaaS system that enables data interoperability by connecting Schema of medical information data of each hospital with the HL7 based standard message, and allowed swift search through the internal category hospital information list of the broker server. This system reduced costs and time by connecting medical data without changing the existing medical information systems. Regarding the Schema collision problem occurring in the process of connecting medical data, XMDR-DAI overcame heterogeneity. Future studies should focus the possibility to enable medical information integration between body clouds by using a sensor.

\section{Acknowledgements}

This article is a revised and expanded version of a paper entitled "A Study on DBaaS System for the HL7-based Health Information Sharing in the Cloud Environment" presented at The $4^{\text {th }}$ International Conference on Information Technology and Computer Science (ITCS 2015) held on July 8-11, 2015 at The Klagan Hotel, Kota Kinabalu, Malaysia.

\section{References}

[1] Eggebraaten Thomas J., J. W. Tenner and J. C. Dubbels, "A health-care data model based on the HL7 reference information model”, IBM Systems Journal, vol. 46, no. 1, (2007), pp.5-18.

[2] "Health Level Seven International (HL7 International)", http://www.hl7.org.

[3] K. H. Singh and K. S. Mann, "Radiology Information System's Mechanisms: HL7-MHS and HL7/DICOM Translation", Computational Intelligence in Data Mining, vol. 1, (2015).

[4] L. Wolfgang and K. U. Sattler, "Database as a service (DBaaS)", Data Engineering (ICDE), 2010 IEEE 26th International Conference on, IEEE, March (2010), pp.1216-1217.

[5] S. Moon, G. D. Jung and Y. K. Choi, "A Study on Cooperation System design for Business Process based on XMDR in Grid", International Journal of Grid and Distributed Computing, vol. 3, no. 3, September (2010), pp. 1-12. 
[6] R. Wesley, "Pragmatic Considerations in the Design of the HL7 Protocol", Proceedings of the Annual Symposium on Computer Application in Medical Care. American Medical Informatics Association, November, (1989), pp. 687-691.

[7] A. S. Oh, "A Study on HL7 Standard Message for Healthcare System Based on ISO/IEEE 11073", International Journal of Smart Home, vol. 9, no. 6, (2015), pp. 113-118.

[8] B. Bernd and L. Seidl, "Concept, Models and Implementations for Innovative Interoperable eHealth Solutions”, EJBI, vol. 11, no. 2, January (2015).

[9] McClay James C., "Standard for improving emergency information interoperability: the HL7 data elements for emergency department systems", Journal of the American Medical Informatics Association, March (2015).

[10] A. Longheu, V. Carchiolo and M. Malgeri, "Medical Data Integration with SNOMED-CT and HL7", New Contributions in Information Systems and Technologies. Springer International Publishing, vol. 353, (2015), pp. 1165-1171.
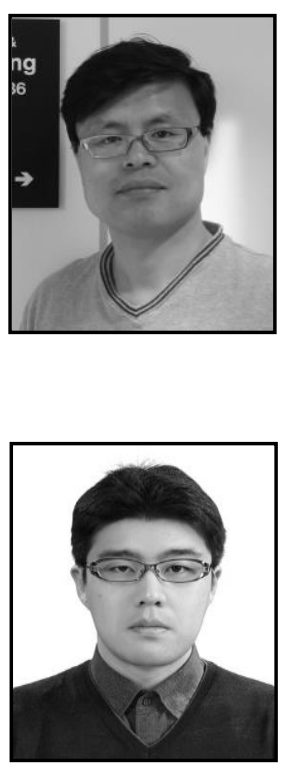

\section{Authors}

Ho-Kyun Park, He received his B.S. and M.S. degrees in computer science from Kwangwoon University, Seoul Korea, in 1987 and 1989, respectively. He obtained his Ph.D. in multimedia network from same university, 1998. He is currently a professor with the School of IT Convergence Engineering at the Shinhan University, Korea, since March 1992. His major research interests include network security, smart home-network system, and ubiquitous computing system.

Seok-Jae Moon, He received the B.S. the M.S. and Ph.D. degrees from Kwangwoon University, Seoul Korea, in 2002, 2004, and 2010, all in computer science. He now works as a Principal Research Engineer in the Department of GlobeBit, Seoul, Korea. He is also an adjunct professor in the School of IT Convergence Engineering at the Shinhan University, Korea. His research interests include big data, business process, cloud computing, data interoperability, and cooperation systems. 
International Journal of Multimedia and Ubiquitous Engineering Vol.10, No.9 (2015) 